\title{
20
}

\section{Comparison of Neural Networks to Ormsbee's Method for Rain Generation - applied to Toronto, Ontario}

Ingmar Wendling and William James

Rainfall rate is a key input function for the analysis and design of hydrologic and hydraulic systems. One common problem with existing records of rain is that the time increments are not fine enough for use in urban storm water models. To solve this problem, observed rainfall data can be disaggregated into shorter time steps.

In this chapter two artificial neural networks are used to disaggregate hourly rainfall data into $5 \mathrm{~min}$ time steps. One model is a multi-layer perceptron (MLP) with a fast back propagation learning algorithm, while the other is a radial basis function (RBF) network with an orthogonal least-squared error-learning algorithm. Both models are described and evaluated.

It is shown that the RBF model performed poorly and its use is not recommended for rainfall disaggregation. However the MLP model achieved generally comparable results to Ormsbee's continuous deterministic model, and did better in the prediction of maximum incremental rainfall depth, but at significantly higher computational effort.

\subsection{Introduction}

In hydrologic modeling, rainfall is the key input variable that activates flow and mass transport. The distribution of rainfall intensity affects runoff, water quality and flood forecasting. The design-storm approach is commonly used

\footnotetext{
Wendling, I. and W. James. 2003. "Comparison of Neural Networks to Ormsbee's Method for Rain Generation - applied to Toronto, Ontario." Journal of Water Management Modeling R215-20. doi: 10.14796/JWMM.R215-20.

(C) CHI 2003 www.chijournal.org ISSN: 2292-6062 (Formerly in Practical Modeling of Urban Water Systems. ISBN: 0-9683681-7-4)
} 
for hydrologic modeling. Synthetic design storms generate single rainfall events for some averaged intensity and duration based on observed storms from a period of record for a certain hydrologic region. However, design storms, at least implicitly, assume certain antecedent conditions (e. g. soil water content, initial detention storage levels), and dry-weather processes are not modeled (James, 1994; Ormsbee, 1989). By using continuous hydrologic modeling, antecedent conditions are addressed directly. To model sustainability issues a duration of simulation between 50 and $100 \mathrm{y}$ may be desirable. The rationale lies in the living memory of environmental changes and catastrophic events, which is passed down through three generations (approx. $75 \mathrm{y}$ ), i.e. when grandparents talk to grandchildren (James, 1993). Be that as it may, long-term continuous modeling is increasingly necessary to deduce the evolution of water quality and the reactions of the aquatic environment to anthropogenic inputs. In this way better decisions can be made to protect the water environment (Yevjevich, 1991).

With today's computer power it is not a problem to compute these longterm simulations. Rainfall data is the major difficulty - in many cases long-term data is available, but the time resolution is too coarse (hourly or daily), whereas time-steps of 5 to $15 \mathrm{~min}$ are needed to run continuous hydrologic models. Data of these small time-steps is also available, but the duration of the record is usually less than $20 \mathrm{y}$. So it will be another fifty-five years before seventy-five years of fine time step historic rainfall data is available (Chan, 1998).

Several rainfall generators have been developed - there are stochastic and deterministic or physically-based models. Stochastic models generate longterm data records by replicating statistical properties of historical rainfall data. Deterministic or physically-based models try to compute rainfall using physical laws and meteorological information.

One way to address this lack of useable data is rainfall disaggregation. Rainfall disaggregation models take historic long-term rainfall data of a coarse time resolution and increase this resolution to produce data with finer time steps. An artificial neural network (ANN) is a powerful tool for solving difficult problems. It can learn the relationship between inputs and the related outputs. After the learning, the learned relationship is encoded in the network. This relationship may be nonlinear and extremely complicated.

Over the last decade neural networks have been applied to solve many problems, including hydrologic problems like streamflow forecasting (Zealand, 1997), urban stormwater analysis (Ingram, 1996) or rainfall runoff modeling (Fernando and Jayawardena, 1998; Furundzic, 1998; Smith and Eli, 1995; Tokar and Johnson, 1999). 


\subsection{Review of Rainfall Disaggregation}

Different rainfall disaggregation models have been developed in the last few decades. Much research has been conducted on the disaggregation of monthly or daily rainfall. These models are not applicable to short-scale rainfall disaggregation.

Hershenhorn and Woolhiser (1987) proposed a disaggregation method for daily rainfall. Their model simulates the number of rainfall events in a day with amount, duration and starting time of each event from daily data. However this method is described as complicated and not appropriate for common engineering purposes (Koutsoyiannis, 1994).

Koutsoyiannis and Xanthopoulos (1990) developed a dynamic disaggregation model for solving stochastic disaggregation problems. Combined with a rainfall model, it is possible to disaggregate monthly rainfall into events and hourly amounts.

Glasbey et al. (1995) used the Rodriguez-Iturbe rainfall model (RodriguezIturbe et al., 1988) to disaggregate daily rainfall into hourly rainfall. Their method is a stochastic point process model that generates artificial rainfall. Point processes are a series of events that occur randomly in time, generating storm origins from a Poisson process. Other researchers also make use of such models for disaggregation (Bo et al., 1994; Cowpertwait et al., 1996a, 1996b; Gyasi-Agyei, 1999).

Other methods concentrate on disaggregating short-scale rainfall for use in continuous hydrologic models.

Ormsbee (1989) developed two empirical disaggregation models. The first is a discrete model that divides hourly values into three 20 -min values. The second is a continuous model that permits disaggregation of hourly rainfall into time intervals from 1 to $30 \mathrm{~min}$. Both models are based on the idea of making the hyetograph of the particular hour geometrically similar to the hyetograph of the precedent, current and successive hours.

Chan (2000) examined long-term short time-interval rainfall generator using a wavelet analysis technique. This technique extracts the spectral behaviour of a historic rain record at various temporal resolutions. A rain reconstruction method is used to produce rainfall in finer time-steps with the observed spectral behaviour. A good prediction of the shape of rainfall was achieved, but unacceptably high annual rainfall characteristics were produced. Burian et al. (2000) were the first to report use of artificial neural network applications for disaggregation of hourly rainfall to fifteen minute rainfall. One model was a feed-forward, three-layer network with a backpropagation 
training algorithm. Another model was also a feed-forward, three-layer ANN using a competitive learning algorithm based on a clustering process. In comparison to other methods these models were deemed successful (Burian et al., undated, personal communication of draft paper, not then published). The need for improvement of the ANN rainfall disaggregation is suggested.

\subsection{Artificial Neural Networks}

Artificial Neural Networks (ANNs) are simplified models of a real nervous system. They simulate biological functions of the human brain. A network consists of interconnected elements, the neurons. These elements are able to respond to an input and adapt to the environment (Patterson, 1996).

The concept of ANN was initiated in the fifties, but at that time the computer power was not developed enough to calculate the complex algorithms. ANNs had their resurrection in the mid eighties and the ANN modeling approach has been used in many applications in different fields in recent years.

A feature of the network is its ability to learn. This procedure consists of giving inputs and matching outputs to the network. It modulates iteratively the internal parameters until the relationship between inputs and outputs is encoded. Then the network is able to produce the desired output from similar inputs (Burian et al., 2001). Hence, difficult problems can be solved by applying information from past experiences.

There are many different architectures of ANNs that can be trained by several types of training algorithms. The most common architecture for engineering problems is a multi-layer feed-forward perceptron (MLP) with backpropagation training algorithm.

ANNs consist of neuron layers: input, hidden and output layers. Two layers of neurons communicate via a weight-connection network. The weight determines the strength of the relationship between the two connected neurons or nodes. The number of nodes in the input and output layer depends on the problem to be solved.

The number and size of the hidden layers is not given. Typically the generalization and accuracy increases as the number of hidden units decreases. As the number of hidden units increases, the network will learn very slowly. This is called the 'principle of generalization versus convergence'.

Many researchers suggest that it is usually unnecessary to use more than one hidden layer in a multi-layer feed-forward network (El-Din and Smith, undated, personal communication, draft paper not then published). Most 
applications of backpropagation use only one hidden layer, because the cells, which are not directly connected to the output layer, will change their weights only a little. Hence, the learning is very slow. But the number of nodes in this layer is critical for the quality of the result. For the best network performance an optimal number of hidden neurons must be properly determined (http:// www.gc.ssr.upm.es/inves/neural/ann1/anntutorial.html, 2001).

An ANN neuron is basically a comparator that produces an output when the cumulative effect of the input stimuli exceeds a threshold value. The confluence or basis function is often the weighted sum of the inputs (variables are defined in the notation at the end of this chapter):

where:

$$
n e t=\sum_{i} v_{j i} x_{i}
$$

$$
\begin{aligned}
x_{i} & =\text { input, and } \\
v_{j i} & =\text { weight. }
\end{aligned}
$$

This is called a first-order (linear) basis function. The activation or mapping function is typically a nonlinear function of the value net. The most common are step, ramp, sigmoid, Gaussian or hyperbolic tangent functions. The neuron is connected by weights to other neurons in adjacent layers. It sends the new calculated information to them.

\subsubsection{The Learning Process}

Learning can be performed in one of these ways:

1. adjusting the weights between neurons,

2. establishing connections between neurons,

3. adjusting the threshold value of activation functions, or

4. combinations of these operations.

There are several learning methods. They can be classified in three basic types: reinforcement, supervised and unsupervised.

In reinforcement learning a teacher has to be present. The network gets an input pattern, but no output. The network is told if the computed output is right or wrong. This way of learning is not very popular.

In supervised learning a teacher also has to be present. The network gets a training input and the matching output. With the difference between input and output pattern an error can be computed. This error can be used to adjust the weights in the network. In unsupervised learning no teacher is present and the network has no information about the output. Therefore, the network must 
learn by discovering and adapting to structured features like statistical regularities or clusterings in the input patterns (Patterson, 1996).

\subsubsection{Multi-layer Feed-Forward Perceptron (MLP) with Fast Backpropagation (FBP) Learning Method}

The first network is a three-layer feed-forward perceptron using a fastbackpropagation-learning method. Only one hidden layer is used.

The input layer consists of three neurons. Inputs are the rainfall amounts of the preceding, actual and succeeding hour. There are twelve neurons in the output layer. Each neuron represents the rainfall depth for $5 \mathrm{~min}$ in the actual hour. The number of hidden nodes must be determined during the training.

The confluence function in hidden and output neurons is the weighted sum of the output of the neurons in the previous layer. The nonlinear activation function is the hyperbolic tangent.

So the calculating process is:

$$
y_{j}=\tanh \left[\beta\left(\sum_{i} x_{i} v_{j i}\right)\right]
$$

in a hidden neuron, and:

in an output neuron.

$$
z_{k}=\tanh \left[\beta\left(\sum_{j} y_{j} w_{k j}\right)\right]
$$

The FBP algorithm is a supervised-learning method. It is based on the reduction of the error function $\mathrm{E}$ using a gradient descent method like the popular backpropagation algorithm. It can be applied to any multilayer network that uses differentiable activation functions.

One drawback of the backpropagation algorithm is that the learning is slow. The weight update is related to the error and the learning rate $\alpha$. During iterations, the output converges to the target. This means the weight updates become smaller, especially the weight updates in the hidden layer. The FBP algorithm accelerates the approach to the error minimum by relating the weight update in the FBP learning method to learning rate and $\varepsilon_{k}(\lambda)$ (Equation 20.7).

At the beginning of the learning $\lambda=1$ which means the second term of Equation 20.7 is zero and the weights are updated by $e_{i}$. During the training, the error decreases and $\lambda$ converges to zero (see Equation 20.12). The second term of Equation 20.7 then becomes dominant and the weights are updated by $\tanh \left(\beta e_{1}\right)$. While the FBP training method can often accelerate the learning 
process, a drawback is that there is a risk that this algorithm will not converge to a minimum error, especially when higher coefficient values are used. For further information see Karayiannis and Venetsanopoulus (1993).

The six-step algorithm of the FBP is:

1. Select $\mu$, set $\lambda=1, \mathrm{E}=0$

2. The output of neurons in the hidden layer and in the output layer are:

$$
\begin{aligned}
& y_{j}=f\left(\sum_{i} x_{i} v_{j i}\right) \\
& z_{k}=f\left(\sum_{j} y_{j} w_{k j}\right)
\end{aligned}
$$

3. Compute the error and weight update:

$$
\begin{aligned}
e_{k} & =z_{k}-t_{k} \\
\varepsilon_{k}(\lambda) & =\lambda e_{k}+(1-\lambda) \tanh \left(\beta e_{k}\right) \\
\Delta w_{k j} & =\alpha \varepsilon_{k} y_{j} \\
\varepsilon_{j}(\lambda) & =\left(1-y_{j}\right)^{2} \sum_{k} \varepsilon_{k} w_{k j} \\
\Delta v_{j i} & =\alpha \varepsilon_{j} x_{i} \\
E & =E+\frac{1}{2} \sum_{k} e_{k}^{2}
\end{aligned}
$$

4. Update all weights and repeat steps 2 and 3 for all training data sets.

5. Compute $\lambda$ :

$$
\lambda=e^{\left(-\mu / E^{2}\right)}
$$

6. Repeat steps $2-5$ until the error has reached an acceptable level.

This program allows the user to select the architecture of a three-layer feed-forward perceptron, like number of neurons in the input, hidden and output layers. 


\subsubsection{Radial Basis Function (RBF) Network with Orthogonal Least Squares (OLS) Algorithm}

RBF networks are becoming increasingly popular with diverse applications and are probably the main rival to the multi-layer perceptron (MLP) (Picton, 2000).

The architecture of a RBF network is similar to that of a MLP network. It also consists of input, hidden and output layers. The input layer is only a fanout layer and does no processing. It sends the input values to the hidden layer. The hidden layer performs non-linear mapping and the output layer performs a simple weighted sum with a linear output. Hidden and output neurons are connected by weights according to their strength.

The number of neurons in the input and output layer depends on the problem solved. The number of hidden nodes is not given before the training, but is determined during the training process. The number of input and output neurons for this network is the same as for the MLP.

The transfer function in the hidden layer neurons is a radially symmetric basis function. The most popular is the Gaussian:

$$
y=\exp \left(-\frac{r^{2}}{2 \sigma^{2}}\right)
$$

If the distance $r$ is zero, the response is equal to one. With a greater distance the response decreases dramatically.

The distance measured from the center $\mu$ is usually the Euclidean distance:

$$
r_{j}=\sqrt{\sum_{i}\left(x_{i}-\mu_{j}\right)^{2}}
$$

So the output of neuron $j$ in the hidden layer is:

$$
y_{j}=\exp \left(-\frac{\sum_{i}\left(x_{i}-\mu_{j}\right)^{2}}{2 \sigma^{2}}\right)
$$

The variable $\sigma$, also called spread, defines the width of the bell shape of the Gaussian function. When the distance from the center reaches $\sigma$ the output drops from 1 to 0.06 .

The output layer calculates the weighted sum for each neuron:

$$
z_{k}=\sum_{k} y_{j} w_{k j}
$$


The parameters in the RBF network are the number of hidden nodes, their RBF functions, and the connection weights between the hidden and output layer. Determining the RBF functions involves finding RBF centers and spreads.

The OLS algorithm, proposed by Chen et al. (1991), adds one hidden unit with the transfer function, which reduces the squared error most, to the hidden layer for every iteration. The training is stopped when a user-defined tolerance is reached. For further information see Chen et al. (1991).

\subsection{Data}

The rainfall data used for this project was gathered from the City of Toronto, City Works Services, Environmental Engineering section. The station identification is Swansea. It is located at 207 Windermere Avenue in Toronto. A tipping bucket was used to collect the data. The data has a time resolution of $5 \mathrm{~min}$ and is recorded in discrete amounts of $0.25 \mathrm{~mm}$. This data is used to create a training and a testing data set (as described below).

The training data for the ANN is the most critical step to eventual success. If the training data is not selected properly, the network will not be able to produce good results. There are some practitioners who state that collecting proper data is nine-tenths of the job (Hammerstrom, 1993).

Rainfall data from $1992-1998$ is used to create the training data set. Hourly rainfall amounts are created by summation of the rainfall amounts during one hour. Then the long-term data record is split into separate storm events. Storm events are separated by at least one hour of no rainfall and the storms have to have a cumulative rainfall depth of at least $1 \mathrm{~mm}$.

Small rainfall events occur more often than large events. If there are more events with small rainfall than events with large amounts of rainfall in the training data sets, the network will only produce good predictions for small events. The results for events with large rainfall amounts will be worse. Therefore, it is important that the training file incorporates an equal number of events with small, average and large amounts of rainfall. In this regard, 260 rainfall events with a total of 1491 data sets were selected for the training file.

The prediction accuracy of a network decreases if data sets with the same input values have different output values. In rainfall disaggregation problems, this can be attributed to (i) different rainfall distributions with the same amount, or (ii) inaccurate recordings of the rainfall amounts. It is prudent to avoid the appearance of these data sets. Therefore, every data set in which input values 
appeared two or more times, were deleted. This reduced the data sets in the training file from 1491 to 1076 .

The recorded rainfalls for the years 1991 and 1999 were used to test the performance of the neural network. The hourly rainfall amounts were calculated in the same manner as for the training file.

The total rainfall depth of the test file was $1238.5 \mathrm{~mm}$ in 164 rainfall events. The file consisted of 794 data sets.

\subsection{Statistics}

20.5.1 Integrated Mean Square Error (IMSE)

where:

$$
\text { IMSE }=\frac{1}{N} \sum_{i=1}^{N}\left(p_{i}^{d}-p_{i}\right)
$$

$$
\begin{array}{ll}
p_{i}^{d}- & \text { disaggregated rainfall depth, and } \\
p_{i}- & \text { observed rainfall depth. }
\end{array}
$$

This statistic is a measure of the area between the generated and observed hyetograph. Small values indicate a good prediction.

20.5.2 Signal-to-Noise Ratio (SN ratio)

$$
S N \text { ratio }=\frac{\sum_{i=1}^{N} p_{i}^{2}}{\sum_{i=0}^{N}\left(p_{i}^{d}-p_{i}\right)^{2}}
$$

The SN ratio describes the size of the signal in comparison to the noise, in this case the size of the observed hyetograph in comparison to the prediction error. High values indicate a good prediction.

\subsection{Determination of Optimum Parameters for the MLP Model}

The learning of the MLP is not only dependent on the training data. Many parameters influence the learning process. The parameters and constants to be determined are: 
Initial weights

Maximum value after standardization

Number of hidden nodes

Steepness of tanh function $(\beta)$

Learning rate $(\alpha)$

Number of data points in the training file

$\mathrm{Mu}(\mu)$

Number of iterations

Order of data points in training file

The training processes were calculated three times for each set of parameters. The results shown are the mean values of those calculations. To compare the performance of each trained network, the integrated mean square error (IMSE) and signal-to-noise ratio (SN ratio) were calculated and plotted.

The chosen parameters for the first simulation are shown in Table 20.1.

Table 20.1 Parameters used for training.

\begin{tabular}{lc}
\hline Number of data sets & 500 \\
Number of iterations & 500 \\
Learning rate $(\alpha)$ & 0.1 \\
Steepness of tanh function $(\beta)$ & 1 \\
$\mathrm{Mu}(\mu)$ & 0.1 \\
\hline
\end{tabular}

\section{Initial Weights}

Initial weights are important for the training process. If the weights are too high, the networks often fail to converge. If the weights are very small, the computations become trapped in local minima and convergence will not be achieved (Patterson, 1996). To prevent this, initial weights with random values within the range of $[-1,1]$ are chosen.

Randomly selected initial weights generate different results for every training, even if all other parameters are constant. For this reason, every training process is done three times and the results presented are the mean values of IMSE and SN ratio.

\section{Maximum Value After Standardization}

The activation function, which is the hyperbolic tangent, permits an input in the range $(-\infty,+\infty)$, but it produces output only in the range $(-1,+1)$. Therefore, the data must be standardized so that possible output values are in this range. In rainfall disaggregation problems, the minimum of the data range is zero, because there are no negative rainfall amounts. 
It is possible that the maximum value is not included in the training data set. To preserve the possibility to predict a value outside of the range of the training data, the maximum training value can be standardized to a value $<1$.

Burian et al. (2001) standardized several training data sets to different values $<1$. Standardizing the maximum value to 0.8 achieved the best results. The 0.8 maximum has been used in other ANN training applications (Smith and Eli, 1995). The following learning processes were performed with a maximum of 0.8 . The program finds the highest value in each column of the training data set and standardizes the column to values between 0 and 0.8 .

\section{Number of Hidden Nodes}

The number of hidden nodes is a critical parameter for the accuracy of the network. If there are too few nodes, the network will fail to converge to a solution. On the other hand, if there are too many hidden neurons, the network will not be able to generalize.

It should be noted that more hidden nodes increase the computation time, because each unit adds to the load on the CPU (central processor unit) during simulations (Freeman et al., 1991). The optimum number of hidden nodes can only be determined by trial and error.

The optimum number of hidden nodes was determined by comparing the performance of the network with 5, 10, 15, 20, 25, 30, 40 and 50 hidden nodes. Unexpectedly, the network with 40 hidden nodes achieved remarkable results. The best performance was achieved by using 15 hidden neurons. This result was verified by an examination of networks with 13, 14, 16 and 17 hidden nodes. The network with 15 hidden nodes showed the best results, so 15 hidden nodes were used in the following training processes.

Beta, $\beta$

The parameter $\beta$ is used in the FBP learning algorithm in the second term of Equation 20.6 for the weight update. It can be used to adjust the steepness of the curve of the hyperbolic tangent function (see Equation 20.19). There are only insignificant changes in the results expected by varying this parameter.

$$
f(x)=\tanh (\beta x)
$$

In this study, the training was executed with values of $0.1,0.5,1,2,4$, 8 and 15 for $\beta$. With a variation of this parameter only insignificant changes in the performance were expected, but the differences are notable. The network 
with a $\beta$ of 4 performed best, so for the following training processes, the steepness of the hyperbolic tangent function was set to 4 .

\section{Learning Rate}

The learning rate $a$ determines the size of the weight updates after each data point during the training process. If $\alpha$ is too high, the error will not converge to the minimum, but will oscillate around it. If $\alpha$ is very small, the weight updates will be very small. This leads to a very slow learning and the training time will expand. The goal is to select a learning rate so that the network is able to converge to a minimum error in an acceptable training time.

In fast backpropagation, the learning rate determines the size of the weight update. The performance of the network was observed with a learning rate of $0.05,0.1,0.15,0.2,0.25$ and 0.5 .

The best performance was achieved with a learning rate of 0.01 . With a higher learning rate, the network is not able to converge to the minimum error, but oscillates around it. The results with a learning rate of 0.5 were unacceptably poor.

The performance decreases with a learning rate below 0.01 . This happens because the weight updates are very small. Hence, the learning is very small and the network does not reach the minimum error after 500 iterations.

The learning rate was set to a value of 0.01 for the following learning processes.

\section{Number of Data Points in Training File}

Generally, the accuracy of the network increases with the quantity of the training data. At a certain point, a larger number of training data sets does not lead to a significant increase or can even lead to a decrease of accuracy. This point, the optimal number of data sets, can only be determined experimentally.

To examine the best number of data points, training files with 250,500 , 750 and 1000 data points were created. The best results were achieved with 1000 data points. Unexpectedly, the network learned worse with 750 data points than with 500. For the following learning processes, the training file with 1000 data points was used.

$M u, \mu$

The parameter $\mu$ determines the parameter $\lambda$, which is important for the weight update (see Equation 20.20).

$$
\lambda=e^{\left(-\mu / E^{2}\right)}
$$


A high $\mu$ means $\lambda$ converges to zero while the error is quite high. When $\mu$ is small, $\lambda$ converges to zero with a smaller error. So $\mu$ is an important parameter for the learning of the network.

Trainings with a $\mu$ of $0.01,0.1,1,10,100,250,500,750$ and 1000 were observed. Surprisingly, the best results were achieved with a $\mu$ of 1 . The error during training never fell below 12. Therefore, $\lambda$ never fell below 0.99 for a $\mu$ with a value of 1 . This means the weights are only updated by the first term of Equation 20.7 during the whole learning process.

\section{Number of Iterations}

The error of the training set and test set typically increases rapidly during the first iterations. The error curves become flatter as the training progresses. Usually the training set error continues to decrease, but the test set error starts to increase at a certain point. After that point, the network starts to learn the noise in the training data. This is called memorizing the training data. The network achieves very good results with the training set, but it loses the ability to generalize on unknown test data.

By testing the network after a different number of iterations, the minimum test set error can be determined.

To find the optimum number of iterations training processes with 50,100 , $250,500,750,1000$ and 2000 iterations were observed. The best results were obtained after 500 simulations. With fewer iterations the performance was worse because the minimum of the error is not reached. After more than 500 iterations the network begins to overfit the data. This leads to a decrease in the ability to generalize.

\section{Different Order of Data Sets in Training File}

The network with the FBP learning algorithm updates the weights after each data set. For this reason training the network with the same data sets in a different order will obtain different results.

The network was trained with three different training files. Each file contains the same data sets, but they are arranged in a different order. Training file $\mathrm{A}$ is the file which was used before. To get file $\mathrm{B}$ and file $\mathrm{C}$ the order of data sets were randomly changed.

Learning with training file $\mathrm{B}$ and $\mathrm{C}$ did not make the network perform better. The best performance was achieved by using training file A. Remarkable is that the performance on the test file with the data of 1991 was almost constant, while the results for the 1999 test file were different. 


\subsection{Determination of Optimum Parameters for the RBF Model}

The OLS learning algorithm determines the number of hidden nodes with the centers of the transfer functions automatically. For this reason, the only parameters to determine for the RBF network are:

- number of data sets,

- spread, and

- tolerance

In contrast to the MLP, the RBF network produced the same results from trainings with the same inputs and parameters. Therefore, a single simulation for each set of parameters is sufficient. The initial settings of the parameters are shown in Table 20.2.

Table 20.2 Initial parameters.

$\begin{array}{cc}\text { Spread }(\sigma) & 0.45 \\ \text { Tolerance }(\mathrm{t}) & 0.1 \\ \end{array}$

\section{Number of Data Sets}

Generally the accuracy of the network increases with a higher number of data sets, but also the number of hidden units will increase. This will cause a complex network computation and longer duration for the learning process.

The accuracy of the network will increase with more data points. One drawback is the computation time. It increases exponentially with the number of data points. For the following learning processes the training data set with 1000 data points was used.

\section{Tolerance}

Tolerance is an input used to stop the training process when the error drops under a certain level. Tolerance is an important parameter in balancing the accuracy and the complexity of the network (Fernando and Jayawardena, 1998). The accuracy improves with increasing complexity of the network.

The training processes with a tolerance of $0.1,0.5,1,2,3,4,5$ and 10 were observed. The best performance was achieved with a tolerance of 1 . This network had 985 neurons in the hidden layer. For the following trainings a tolerance of 1 was selected. 


\section{Spread}

The spread $\sigma$ defines the width of the bell shape of the Gaussian function. If this variable is too small, the network will only give accurate results for inputs which are part of the training data. It will produce very small results for unknown data, and will hardly be able to interpolate.

The spread is the most important parameter for the accuracy of the network. With a small spread, IMSE and SN ratio show better results, but the total volume of predicted rainfall is too small.

Table 20.3 shows that with a large spread of the Gaussian function, the volume of the predicted rainfall is too large. With a spread of 0.51 the volume of predicted rainfall is nearest to the volume of the observed rainfall.

Table 20.3 Volume of predicted rainfall (in \% of observed rainfall).

\begin{tabular}{cl}
\hline$\sigma$ & Test Data \\
\hline 0.45 & $81.5 \%$ \\
0.47 & $87.4 \%$ \\
0.49 & $92.7 \%$ \\
0.51 & $100.2 \%$ \\
0.53 & $107.7 \%$ \\
\hline
\end{tabular}

\subsection{Results}

To evaluate the performance of the two neural network models, the test data was disaggregated with Ormsbee's continuous deterministic model (Ormsbee, 1989). The MLP network with the best performance and the RBF network, which produces about $100 \%$ of the rainfall for the test data, were chosen for comparison.

IMSE, SN ratio and percent volume of the predicted rainfall are shown in Table 20.4. Recall that a lower IMSE and a higher SN ratio reflects a better prediction.

Table 20.4 Performance of the models.

\begin{tabular}{llll}
\hline Model & IMSE & SN ratio & Volume \\
\hline MLP & 0.0803 & 1.6516 & $95.7 \%$ \\
RBF & 0.1738 & 0.7635 & $100.2 \%$ \\
Ormsbee & 0.0791 & 1.6730 & $100.0 \%$ \\
\hline
\end{tabular}


The performance of the RBF network is poor compared to the other models. IMSE and SN ratio indicate that the MLP and the Ormsbee model perform similarly. For the 1991 test data set, the Ormsbee model achieved better results and for the 1999 data set the MLP model performed insignificantly better.

For hydrologic modeling, the peak intensity of the rainfall during an event is important because it has significant influence on the resulting runoff hydrograph. The Ormsbee model predicts the peak rainfall intensity with a mean underprediction of $53 \%$. With a mean underprediction of $47 \%$ the MLP model is somewhat better, but still significantly too low. The RBF network predicted the peak intensity with a mean overprediction of $55 \%$.

\subsection{Conclusion}

Two ANN models were introduced and evaluated in this study. One network was a multi-layer feed-forward perceptron with a fast backpropagation learning algorithm. The other model was a radial basis function network with an orthogonal least squared error learning algorithm. The performance of both networks was compared to a continuous deterministic disaggregation model.

- The performance evaluation indicated that the RBF network performed poorly compared to the other models.

- The MLP model performed similarly to the Ormsbee model. This network model provided a better prediction of the peak rainfall intensity during the rainfall events than did the Ormsbee model. This was also observed in previous studies (Burian et al., 2000).

- The MLP is a practicable alternative to other models, but it is time consuming for the hydrologist to create a training file and to determine the optimum parameters.

- The RBF network produced very poor results and on this evidence cannot be recommended for rainfall disaggregation.

\section{Notation}

$\begin{array}{ll}\alpha & \text { learning rate } \\ \beta & \begin{array}{l}\text { parameter to adjust the steepness of the hyperbolic } \\ \text { tangent function }\end{array} \\ \varepsilon_{k} & \text { error, calculated to update the weights in FBP }\end{array}$




$\lambda$
$\mu$
$\sigma$
$\mathrm{c}$
$\mathrm{e}_{\mathrm{k}}$
$E$
$i$
$j$
$k$
$r$
$t$
$t a n h$
$v_{j i}$
$w_{k j}$
$x_{i}$
$y_{j}$
$z_{k}$

value to calculate weight update in FBP value to calculate $\lambda$ in FBP spread in the Gaussian function center of the Euclidean distance error between observed and predicted output of node $\mathrm{k}$ of the output layer summed squared error node $i$ of input layer node $\mathrm{j}$ of hidden layer node $\mathrm{k}$ of output layer radius tolerance hyperbolic tangent function weight between node $i$ of input layer and node $j$ of hidden layer weight between node $j$ of hidden layer and node $k$ of output layer input value of node $i$ of the input layer output value of node $j$ of the hidden layer output value of node $\mathrm{k}$ of the output layer

\section{References}

Bo, Z., Islam, S. and Eltahir, E. A. B. 1994. Aggregation-disaggregation properties of a stochastic rainfall model. Water Resources Research. 30(12): 3423-3435.

Burian, S. J., Durrans, S. R., Tomic, S., Pimmel, R. L. and Ngai Wai, C. 2000. Rainfall Disaggregation Using Artificial Neural Networks. Journal of Hydrologic Engineering. 5(3): 299-307.

Burian, S. J., Durrans, S. R., Nix, S. J. and Pitt, R. E. 2001. Training Artificial Neural Networks to Perform Rainfall Disaggregation. Journal of Hydrologic Engineering. 6(1): 43-51.

Burian, S. J. and Durrans, S. R. undated Short Time-Interval Rainfall Disaggregation for Continuous Hydrologic Simulation. Draft paper not yet published.

Chan, A. 2000. "Wavelet Techniques for the Analysis and Synthesis of Rainfall Data." Journal of Water Management Modeling R206-08. doi: 10.14796/JWMM.R206-08.

Chen, S., Cowan, C. F. N. and Grant, P. M. 1991. Orthogonal Least Squares Learning Algorithm for Radial Basis Functions Networks. IEEE Transactions on Neural Networks. 2 (2): 302-309. 
Cowpertwait, P. S. P., O’Connell, P. E., Metcalfe, A. V. and Mawdsley, J. A. 1996a. Stochastic point process of rainfall. I. Single-site fitting and validation. Journal of Hydrology. 175: 17-46.

Cowpertwait, P. S. P., O'Connell, P. E., Metcalfe, A. V. and Mawdsley, J. A. 1996 b. Stochastic point process of rainfall. II. Regionalisation and disaggregation. Journal of Hydrology. 175: 47-65.

El-Din, A. G. and Smith, D. W. undated Forecasting Wastewater Inflow using an Artificial Neural Network. draft paper not yet published.

Fernando, D. A. K. and Jayawardena, A. W. 1998. Runoff forecasting using RBF networks with OLS algorithm. Journal of Hydrologic Engineering. 3 (3): 203-209.

Furundzic, D. 1998. Application example of neural networks for time series analysis: Rainfall-runoff modeling. Signal Processing. 64: 383-396.

Glasbey, C. A., Cooper, G. and McGechan, M. B. 1995. Disaggregation of daily rainfall by conditional simulation from a point process model. Journal of Hydrology. 165: 1-9.

Gyasi-Agyei, Y. 1999. Identification of regional parameters of stochastic model for rainfall disaggregation. Journal of Hydrology. 223: 148-163.

Hammerstrom, D. 1993. Working with Neural Networks. IEEE Spectrum. July: 46-53.

Hersenhorn, J. and Woolhiser, D. A. 1987. Disaggregation of Daily Rainfall. Journal of Hydrology. 95(3): 299-322.

http://www.gc.ssr.upm.es/inves/neural/ann1/anntutorial.html, 2001. Artificial Neural Networks. World Wide Web Page. Escuela Técnica Superior de Ingenieros de Telecomunicación. Universidad Politécnica de Madrid. Last Accessed: August $21,2001$.

Ingram, M. R. 1996. An assessment of three neural network applications for an urban stormwater pond in Kingston Township. M. Sc. thesis, Queen's University. Kingston, Ontario.

James, W. 1994. On reasons why traditional single-valued, single-event hydrology (typical design storm methodology) has become simple-minded, dishonest and unethical. Urban Hydrology \& Hydraulics Workshop. US Army Corps of Engineers Hydrologic Engineering Center, Davis, California. September 13-15.

James, W. 1993. On the need for three-generation continuous models of stormwater impacts. USEPA Invited Conference on Model-building for Great Lakes Water Quality problems of the 2000's. Heidelberg College, Tiffin, OH. August 4-6.

Karayiannis, N. B. and Venetsanopoulos, A. N. 1993. Artificial Neural Networks Learning Algorithms, Performance Evaluation, and Applications. Kluwer Academic Publishers.

Koutsoyiannis, D. 1994. A Stochastic Disaggregation Method for Design Storm and Flood Synthesis. Journal of Hydrology. 156: 193-225.

Koutsoyiannis, D. and Xanthopoulos. 1990. Dynamic Model for Short-Scale Rainfall Disaggregation. Hydrological Sciences Journal. 35(3): 303-322.

Ormsbee, L. E. 1989. Rainfall Disaggregation Model for Continuous Hydrologic Modeling. Journal of Hydraulic Engineering. 115(4): 507-525. 
Patterson, D. W. 1996. Artificial Neural Networks - Theory and Applications. Prentice Hall Simon \& Schuster (Asia) Pte Ltd., Singapore.

Picton, P. 2000. Neural Networks. Palgrave, New York.

Rodriguez-Iturbe, I., Cox, D. R. and Isham, V. 1988. A point process model for rainfall: further developments. Proc. R. Soc. London. A 417: 283-298.

Smith, J. and Eli, R. N. 1995. Neural Network Models of Rainfall-Runoff Process. Journal of Water Resources Planning and Management. 121 (6): 499-508.

Tokar, A. S. and Johnson, P. A. 1999. Rainfall-Runoff Modeling using Artificial Neural Networks. Journal of Hydrologic Engineering. 4(3): 232-239.

Yevjevich, V. 1991. Tendencies in Hydrology Research and Its Applications for 21st Century. Water Resources Management. 5 (1): 1-23.

Zealand, C. M. 1997. Short term streamflow forecasting using artificial neural networks. M. Sc. thesis, University of Manitoba. Winnipeg, Manitoba. 